\title{
Activation of p38 mitogen-activated protein kinase module facilitates in vitro host cell invasion by Rickettsia rickettsii
}

Interactions with target membrane molecules and the resultant internalization into host cells are, in general, the first and foremost steps for subsequent host responses and pathogenesis of infectious micro-organisms. For pathogenic bacteria belonging to both spotted fever and typhus subgroups of Rickettsia species, which are known for dependence on the nutrientrich environment of the host cytoplasm and fastidious growth requirements, target cell invasion is absolutely critical for subsequent intracellular replication and intercellular spread. Rickettsia rickettsii, the causative agent of Rocky Mountain spotted fever, primarily affects the endothelial cell lining of small- and medium-sized vessels leading to disseminated intracellular infection of the vasculature and, consequently, characteristic pathological features during human infections correspond to vascular inflammation, damage and dysfunction (Walker, 2007).

Initial studies using Rickettsia prowazekii, the aetiological agent of epidemic typhus, revealed that inactivation of bacteria or alterations in the host cell cytoskeleton adversely affect rickettsial uptake into cultured human umbilical vein-derived endothelial cells, suggesting that the viability of both the host cell and adherent rickettsiae is an essential prerequisite for successful invasion via 'induced phagocytosis' (Walker, 1984). Subsequent investigations by electron microscopy further indicated that rickettsial entry into Vero cells is almost instantaneous (occurring within 3 min of bacteriumhost cell contact) and rickettsiae are able to quickly escape from the phagosome into the cytoplasm, presumably prior to phagolysosomal fusion (Teysseire et al., 1995), and likely via a phospholipase activity (Whitworth et al., 2005). An important role for host actin polymerization in rickettsial internalization of non-phagocytic cells has also been suggested. Analysis of proteins known to govern actin dynamics has identified recruitment of the Arp2/3 complex to the sites of entry foci during Rickettsia conorii invasion of Vero cells, and potential coordinated involvement of GTPase Cdc42, phosphoinositide 3-kinase, $\mathrm{c}$-Src and other protein tyrosine kinase activities in the activation of the Arp $2 / 3$ complex (Martinez \& Cossart, 2004).

Conserved across all eukaryotes, the p38 group of mitogen-activated protein (MAP) kinases is implicated in many physiological processes, including cell growth, differentiation, apoptosis and immune response. A unique feature for activation of the p38 superfamily of enzymes is the requirement for dual phosphorylation of the TXY motif in the activation loop of the catalytic domain, where phosphorylation of tyrosine $(\mathrm{Y})$ usually precedes that of threonine $(\mathrm{T})$ in a two-step reaction. Our laboratory has demonstrated that in vitro infection of cultured human endothelial cells with spotted fever rickettsiae ( $R$. rickettsii and $R$. conorii) triggers the activation of the p38 signalling module, the specific inhibition of which results in downregulation of infection-induced expression of chemokines [interleukin-8 (CXCL8) and monocyte chemoattractant protein-1 (CCL-2)], and downregulation of the inducible isoform of regulatory bifunctional enzyme cyclooxygenase-2 (Rydkina et al., 2005, 2006). In this report, we have further investigated the possibility of an interrelationship between p38 MAP kinase activation and $R$. rickettsii invasion of host endothelial cells.

Primary cultures of endothelial cells isolated from the umbilical vein of freshly collected human umbilical cords were established and maintained according to previously described procedures (Sahni et al., 1998). Vero C1008 cells were obtained from American Type Culture Collection and maintained in culture as recommended by the supplier. The Sheila Smith strain of $R$. rickettsii was grown in Vero cells and purified on sucrose-
Renografin density gradients (Sahni et al., 1998; Rydkina et al., 2005). The pyridinyl imidazole SB 203580 was used as a selective inhibitor of p38 MAP kinase. This compound does not inhibit other MAP kinase homologues c-Jun-N-Kinase (JNK) and p42 MAP kinase, but has previously been shown to specifically inhibit $R$. rickettsii-induced activation of p38 (Rydkina et al., 2005). SB 202474, a structurally similar compound with no inhibitory activity towards p38 activity, was used as a negative control in all experiments. To determine the extent of rickettsial invasion into host cells, we employed three independent approaches: immunofluorescent staining of rickettsiae followed by quantification of the percentage of cells infected and the number of intracellular bacteria (Sahni et al., 1998), plaque formation assay (Rydkina et al., 2005) and citrate synthase gene ( $g l t A$ )-based real-time PCR (Rydkina et al., 2007). Plaque formation assays were carried out using Vero cell monolayers, whereas indirect immunofluorescence and quantitative PCR-based enumeration was conducted using human endothelial cells. The durations for inhibitor treatment as well as $R$. rickettsii infection for both cell types were identical; each experimental condition was repeated to achieve a minimum of three replicates, and results obtained were subjected to statistical evaluation as described previously (Rydkina et al., 2005, 2007).

First, we compared the level of infection in endothelial cells grown to confluence on sterile coverslips and incubated for $3 \mathrm{~h}$ with $R$. rickettsii in the presence and absence of $10 \mu \mathrm{M}$ SB 203580 (SB 80) and $10 \mu \mathrm{M}$ SB 202474 (SB 74) in the culture medium. Indirect immunofluorescent staining using an anti- $R$. rickettsii antiserum and a rhodamine-conjugated compatible secondary antibody revealed that in the presence of specific p 38 inhibition via SB 80 the number of adherent/intracellular rickettsiae was 
comparatively lower than in those endothelial cells infected in culture medium alone or in the presence of SB 74, an inactive structural analogue of SB 80 (Fig. 1). The extent of plaque formation on confluent monolayers of Vero cells was next determined under similar experimental conditions. After $3 \mathrm{~h}$ exposure to viable $R$. rickettsii, which was followed by thorough washing and layering of the cell monolayer with $0.5 \%$ agarose in culture medium, visibly distinct plaques resulted due to infection-induced lysis of host cells on day 7 post-infection. Again, cell treatment with SB 74 during the process of initial contact and invasion had no effect on the ability to induce plaque formation. Presence of SB 80 during infection, however, led to a significant decrease $(36 \pm 7 \%)$ in the number of rickettsial plaques $(P=0.017$, when compared to corresponding untreated controls and SB 74-treated cells) (Fig. 2).

Next, the number of intracellular rickettsiae in endothelial cells infected in the presence of p38 inhibition was quantified by a real-time PCR-based approach using a rickettsial $g l t A$-specific primer pair and a TaqMan probe to determine the mean copy number of glt $A$ in relation to that of the housekeeping gene GAPDH. Again, treatment of endothelial cells with SB 80 significantly reduced the invasion of $R$. rickettsii $(\sim 38 \pm 12 \%$ inhibition in comparison to cells infected without any treatment; $P=0.025)$. Infection in the presence of SB 74 , a negative control for SB 80 , had no significant effect on rickettsial invasion when compared to controls $(\sim 17 \pm 13 \%$ inhibition; $P=0.21$ ) (Fig. 2). Thus, when directly compared to infection in the presence of SB 74, SB 80 caused an approximately $25 \%$ decrease in the invasion of $R$. rickettsii. Taken together, these results suggest that p38 MAP kinase activation in response to infection plays an important role in facilitating host cell invasion by $R$. rickettsii.

As pathogenic bacteria with an obligate intracellular lifestyle, internalization by host cells, followed by replication within the cytoplasm, has important implications in the establishment, progression and dissemination of infection for various Rickettsia species. It is well known that among major immunodominant surface antigens, rickettsial outer membrane protein $\mathrm{A}^{-}(\mathrm{rOmpA})$ and $\overline{\mathrm{rO}} \mathrm{mpB}$ are expressed by spotted fever group rickettsiae. Inhibition studies using mAbs against both of these proteins are clearly suggestive of a critical role for rOmpA in $R$. rickettsii adhesion to host $\mathrm{L} 929$ cells ( $\mathrm{Li}$ \& Walker, 1998). More recent proteomic characterization of rickettsial adhesins by two-dimensional electrophoresis coupled with MS analysis has revealed two highly conserved, putative rickettsial ligands, one of which was identified as a C-terminal $\beta$ peptide of $\mathrm{rOmpB}$, whereas the other was a protein of unknown function encoded by ORFs RC1281 in R. conorii and RP828 in $R$. prowazekii (Renesto et al., 2006). Interestingly, interactions between $\mathrm{rOmpB}$ and Ku70, a DNA binding component of DNA-dependent protein kinase, have recently been implicated in $R$. conorii entry into non-phagocytic host cells (Martinez et al., 2005). The potential contribution of host cell signalling mechanisms in facilitating internalization of rickettsiae, however, remains relatively poorly defined. $R$. conorii uptake into mammalian cells has been shown to be associated with tyrosinespecific phosphorylation of a number of host proteins, including focal adhesion kinase, and efficient invasion is dependent not only on actin polymerization, but also on activities of phosphoinositide 3-kinase

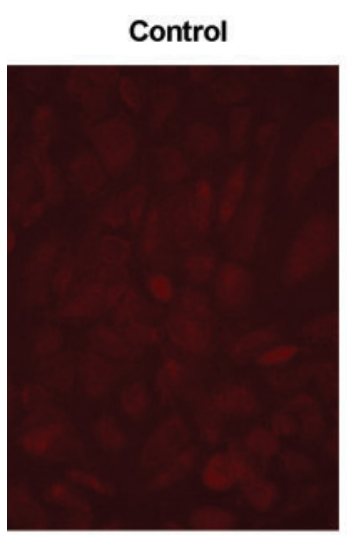

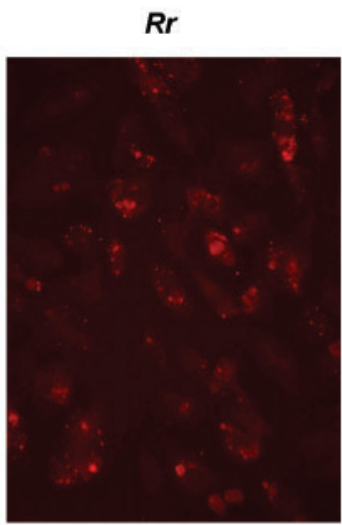

$(7.0 \pm 1.7)$

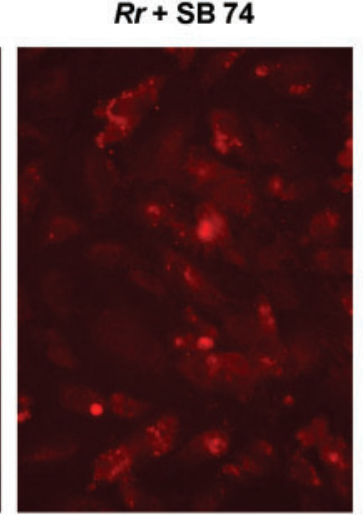

$(4.9 \pm 1.5)$ $P=0.31$
$R r+\mathrm{SB} 80$

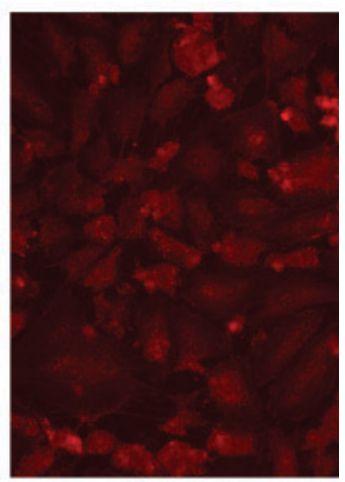

$(3.1 \pm 1.1)$

Fig. 1. Immunofluorescent staining for $R$. rickettsii $(R r)$ in endothelial cells infected in the presence and absence of specific p38 kinase inhibitor SB 203580 (SB 80) or its inactive structural analogue SB 202474 (SB 74). Cells grown to $\geqslant 80 \%$ confluence on sterile plastic coverslips were treated with SB 80 or SB 74 at a final concentration of $10 \mu \mathrm{M}$ for 30 min prior to and during $3 \mathrm{~h}$ infection with $R$. rickettsii. After fixation, coverslips, including those with uninfected endothelial cells (control), were incubated with an anti- $R$. rickettsii primary antibody followed by a rhodamine-conjugated secondary antibody. The level of intracellular infection was then determined by counting a minimum of 300 infected cells with or without SB 74/SB 80 treatment. Representative photomicrographs for each experimental condition are shown. Figures in parentheses underneath the images represent the number of intracellular rickettsiae per infected cell, calculated as the mean $\pm \mathrm{SE}$ from three separate experiments. As determined by Student's $t$-test, $P$ values for cells treated with SB 74 or SB 80 in relation to infection alone are also shown. 


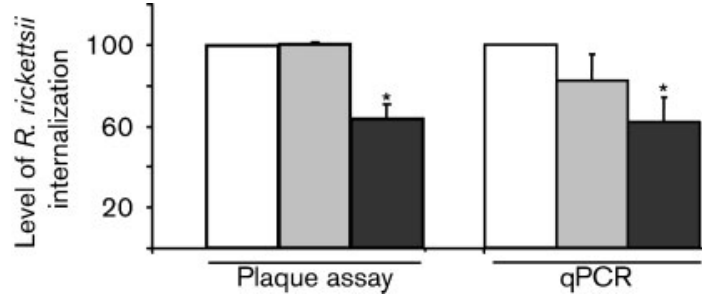

Fig. 2. Effect of selective p38 kinase inhibition on internalization of $R$. rickettsii into host cells. Vero $\mathrm{C} 1008$ and endothelial cells were either left untreated or incubated for 30 min with SB 74 and SB 80, after which infection was carried out for $3 \mathrm{~h}$ in the continued presence of inhibitor compounds. The level of rickettsial invasion was then determined by plaque formation assay in Vero cells and by real-time quantitative PCR (qPCR) in endothelial cells. The number of internalized rickettsiae in cells receiving no treatment (white bars) was assigned an absolute value of 100 and compared with cells infected with $R$. rickettsii in the presence of SB 74 (grey bars) or SB 80 (black bars). The data are presented as the mean \pm SEM from at least three independent experiments. Asterisks indicate a statistically significant decrease in comparison to infection alone $(P \leqslant 0.05)$.

and protein tyrosine kinase (Martinez \& Cossart, 2004).

Three major MAP kinase cascades, namely extracellular signal-regulated kinases (ERKs), c-Jun-N-terminal kinases and p38 MAP kinases, have been identified and characterized in detail. These subfamilies can be activated simultaneously or independently in a variety of situations to constitute a critical component of a 'central' regulatory mechanism. Involvement of MAP kinases in host cell responses to infection with a number of pathogenic bacteria has been established (Rydkina et al., 2005). Available evidence further implicates MAP kinases in the regulation of cytoskeletal organization in various systems, including invasion of host epithelial cells by Campylobacter jejuni and other invasive enteric bacterial pathogens ( $\mathrm{Hu}$ et al., 2006). Similarly, uptake of Chlamydia pneumoniae by HEp-2 cells is effectively blocked by U0126, a specific inhibitor of MEK1/2-dependent activation of ERK1/2 (Coombes \& Mahony, 2002). Further, interaction of Neisseria meningitidis with human brain microvascular endothelial cells results in phosphorylation and activation of JNK1 and JNK2 and p38 MAP kinase, as well as their direct substrates cJun and MAP kinase-activated kinase-2, respectively. In this case, inhibition of JNK via SP600125 reduces the invasion of meningococci, whereas p38 inhibitors
SB202190 and SB203580 have no effect (Sokolova et al., 2004).

Using in vitro infection of cultured human endothelial cells, the preferred target cell type in patients with confirmed diagnosis of spotted fever rickettsioses and in established in vivo models of infection, we have shown selective activation of p38 MAP kinase during infection with $R$. rickettsii and $R$. conorii (Rydkina et al., 2005, 2007). The present communication provides what is believed to be the first evidence that $R$. rickettsii internalization into endothelial cells likely involves signalling through the p38 module of MAP kinases. This observation corroborates our earlier findings that adherence of viable rickettsiae to the host cell surface is apparently sufficient to initiate intracellular signalling events to induce $\mathrm{p} 38$

phosphorylation and activation (evident as early as 15 min after contact between endothelial cells and $R$. rickettsii). Such an immediate-early response, in turn, facilitates the process of bacterial internalization into host cells. It is important to emphasize, however, that partial blockade of invasion in the presence of $\mathrm{p} 38$-specific inhibition suggests the participation of multiple signalling mechanisms, and supports the possibility of rickettsial interactions with other cell surface receptors in addition to Ku70 (Martinez et al., 2005). Acquiring detailed knowledge of signalling pathways exploited by pathogenic Rickettsia species to invade different mammalian cells should not only allow better understanding of host tissue tropism, but should also lead to the development of novel strategies aimed at preventing invasion as the fundamental pathogenesis mechanism.

\section{Acknowledgements}

We thank Semion Kiriakidi for excellent technical assistance. This research was supported in part by United States Public Health Service grants AI 040689 and AI 067613 from the National Institute of Allergy and Infectious Diseases of National Institutes of Health, Bethesda, MD, USA.

\section{Elena Rydkina, Loel C. Turpin and Sanjeev K. Sahni}

\section{Department of Microbiology and Immunology, University of Rochester School of Medicine and Dentistry, Rochester, NY 14642, USA}

Correspondence: Sanjeev K. Sahni (Sanjeev_Sahni@urmc.rochester.edu)

Coombes, B. K. \& Mahony, J. B. (2002) Identification of MEK- and phosphoinositide 3-kinase-dependent signalling as essential events during Chlamydia pneumoniae invasion of HEp2 cells. Cell Microbiol 4, 447-460.

Hu, L., McDaniel, J. P. \& Kopecko, D. J. (2006). Signal transduction events involved in human epithelial cell invasion by Campylobacter jejuni 81-176. Microb Pathog 40, 91-100.

Li, H. \& Walker, D. H. (1998). rOmpA is a critical protein for the adhesion of Rickettsia rickettsii to host cells. Microb Pathog 24, 289-298.

Martinez, J. J. \& Cossart, P. (2004). Early signaling events involved in the entry of Rickettsia conorii into mammalian cells. J Cell Sci 117, 5097-5106.

Martinez, J. J., Seveau, S., Veiga, E., Matsuyama, S. \& Cossart, P. (2005). Ku70, a component of DNA-dependent protein kinase, is a mammalian receptor for Rickettsia conorii. Cell 123, 1013-1023.

Renesto, P., Samson, L., Ogata, H., Azza, S., Fourquet, P., Gorvel, J. P., Heinzen, R. A. \& Raoult, D. (2006). Identification of two putative rickettsial adhesins by proteomic analysis. Res Microbiol 157, 605-612.

Rydkina, E., Silverman, D. J. \& Sahni, S. K. (2005). Activation of $\mathrm{p} 38$ stress-activated protein kinase during Rickettsia rickettsii infection of human endothelial cells: role in the induction of chemokine response. Cell Microbiol 7, 1519-1530.

Rydkina, E., Sahni, A., Baggs, R. B., Silverman, D. J. \& Sahni, S. K. (2006). Infection of human 
endothelial cells with spotted fever group rickettsiae stimulates cyclooxygenase 2 expression and release of vasoactive prostaglandins. Infect Immun 74, 5067-5074.

Rydkina, E., Sahni, A., Silverman, D. J. \& Sahni, S. K. (2007). Comparative analysis of host-cell signalling mechanisms activated in response to infection with Rickettsia conorii and Rickettsia typhi. J Med Microbiol 56, 896-906.

Sahni, S. K., Van Antwerp, D. J., Eremeeva, M. E., Silverman, D. J., Marder, V. J. \& Sporn,

L. A. (1998). Proteasome-independent activation of nuclear factor- $\kappa \mathrm{B}$ in cytoplasmic extracts from human endothelial cells by Rickettsia rickettsii. Infect Immun 66, 1827-1833.

Sokolova, O., Heppel, N., Jägerhuber, R., Kim, K. S., Frosch, M., Eigenthaler, M. \& Schubert-Unkmeir, A. (2004). Interaction of Neisseria meningitidis with human brain microvascular endothelial cells: role of MAPand tyrosine kinases in invasion and inflammatory cytokine release. Cell Microbiol 6, 1153-1166.

Teysseire, N., Boudier, J.-A. \& Raoult, D. (1995). Rickettsia conorii entry into Vero cells. Infect Immun 63, 366-374.
Walker, T. S. (1984). Rickettsial interactions with human endothelial cells in vitro: adherence and entry. Infect Immun 44, 205-210.

Walker, D. H. (2007). Rickettsiae and rickettsial infections: the current state of knowledge. Clin Infect Dis 45, S39-S44.

Whitworth, T., Popov, V. L., Yu, X. J., Walker, D. H. \& Bouyer, D. H. (2005). Expression of the Rickettsia prowazekii pld or $t l y C$ gene in Salmonella enterica serovar Typhimurium mediates phagosomal escape. Infect Immun 73, 6668-6673. 